\title{
Clinico-Metabolic Profile of Neurometabolic Disorders of Children in a Tertiary Care Hospital of Bangladesh
}

\author{
G.K. Kundu, R. Islam, and S. Ahmed
}

\section{ABSTRACT}

Background: Neurometabolic disorders are inborn errors of metabolism with neurological manifestations. They are individually rare but as a group have a significant burden. They constitute $4.9 \%$ of genetic cause of moderate and severe mental retardation.

Aims and objective: To explore clinical \& metabolic profile of various forms of childhood Neurometabolic disorders.

Methods: A hospital based retrospective study was conducted at Department of Pediatric Neurology, Bangabandhu Sheikh Mujib Medical University, and Dhaka from January 2016 to December 2020. Total 59 suspected children of Neurometabolic disorders (NMD), who admitted in hospital during the study period, were included. Among them 41 cases of NMD were diagnoses confirmed on the basis of clinical suspicion, biochemical test, and neuroimaging study and in few cases molecular genetics investigations.

Results: Among 59 suspected cases, total 41 patients were diagnosed as Neurometabolic disorders. Most of the $(63.2 \%)$ patients were within $0-3$ years age. Male patients $\mathbf{( 7 0 . 7 3 \% )}$ were outnumbered than female (29.26\%). Male: Female ratio were 2.4:1. More than half of patients $25(60.90 \%)$ had parental consanguinity followed by Sib death 7 $(\mathbf{1 7 . 0 7 \%})$, Prematurity 11 (26.82\%). Common Neurometabolic disorders were aminoacidopathy $14(34.14 \%)$ and lysosomal storage disorders 15 $(36.58 \%)$ followed by mitochondrial disorders $5(12.19 \%)$, Urea cycle disorders 3(7.31\%), CHO metabolism disorders 5(2.43\%), Peroxisomal disorders $3(7.31 \%)$. Among aminoacidopathy, Organic acidamia 4(9.72\%), MSUD 3(7.31\%), PKU 2(4.86\%), Gluteric Aciduria 3(7.31\%), and Biotinidase deficiency disorder $\mathbf{1}(2.43 \%)$ were common disorders. Among lysosomal storage disorders, mucopolysaccaridosis $4(9.72 \%)$ and metachromatic leukodystrophy $8(19.44 \%)$ were most common disorders. Common presentations were developmental delay $37(90.24 \%)$, developmental regression $21(51.21 \%)$, hypotonia $29(70.73)$ and seizures $26(63.41 \%)$. Neuroimaging changes found in about three fourth $(\mathbf{8 0 . 4 9 \%})$ of cases. About $\mathbf{2 6 . 8 2 \%}$ cases presented with white matter hyper intensity followed by basal ganglia hyper intensity $(\mathbf{1 9 . 5 1 \%})$ and cerebral atrophy $(\mathbf{1 4 . 6 3 \%})$. Abnormally high ammonia and lactate found in $6(14.63 \%)$ and $10(24.40 \%)$ cases respectively followed by Positive urinary ketones 8 (19.51\%).

Conclusions: Neurometabolic disorders are not uncommon. The commonest neurometabolic disorders were aminoaciduria and lysosomal storage disease in our study. Among aminoacid disorders, Organic acidamia, MSUD, Glutaric aciduria and in lysosomal storage disorders metachromatic leukodystrophy \& Mucopolysaccharoidosis were common disorders. Neuroimaging changes were found in about three- forth cases in this study.

Keywords: Clinical profile, neurometabolic disease.
Published Online: August 10, 2021

ISSN: 2736-5476

DOI: $10.24018 /$ ejclinicmed.2021.2.4.105

\section{G.K. Kundu*}

Department of Paediatric Neurology, Bangabandhu Sheikh Mujib Medical University (BSMMU), Dhaka, Bangladesh.

(e-mail: gopen.kundu@gmail.com)

R. Islam

Resident, Department of Paediatric Neurology, Bangabandhu Sheikh Mujib Medical University (BSMMU), Dhaka, Bangladesh.

(e-mail: irumana43@gmail.com)

S. Ahmed

Consultant, Department of Paediatric Neurology, Bangabandhu Sheikh Mujib Medical University (BSMMU), Dhaka, Bangladesh.

(e-mail: sanjida.lipi@yahoo.com)

*Corresponding Author

\section{INTRODUCTION}

Neurometabolic disorders (NMD) are inborn errors of metabolism with neurological manifestations. Individually rare but as a group NMD have a significant burden in worldwide. Neurometabolic disorders, an inherited conditions refer to a group of disorders that are characterized by a lack or dysfunction of an enzymes or vitamins necessary for a specific chemical reaction in the body [1]. Over one third of the inherited metabolic disorders are characterized by the 
central nervous system involvement [2].

These disorders result in substrate accumulation causing minor to severe neurological and psychiatric manifestations and resulting in lifelong disability or death. The incidence of IEM collectively is estimated to be as high as 1 in 800 live births, but it varies greatly [3]. Constitute $4.9 \%$ of genetic causes of moderate and severe mental retardation.

Progressive encephalopathy with psycho-sensory motor regression such as hypotonia, developmental delay, seizures of unexplained cause, recurrent episodes of confusion or coma, severe metabolic acidosis, persistent vomiting and peculiar odor, parental consanguinity, siblings or relatives with a similar history or unexplained neurological syndrome or deaths, progressive gait dysfunction, progressive cerebellar ataxia are the common menufestations [3].

The most common neurometabolic disorders to be considered are amino acid apathies followed by neuronal ceroid lipofuscinoses, urea cycle disorders, congenital lactic acidosis, peroxisomal disorders, and, less frequently, sphingolipidoses, mucopolysaccharidoses, glycoprotein degradation disorders and fatty acid oxidation disorders [4], [5].

There is no such type of study of neurometabolic disorder in Bangladesh. Therefore, we want to explore clinical \& metabolic profile of various forms of Neurometabolic disorders in our tertiary care hospital.

\section{Methodology}

This hospital based retrospective study was done to observe to explore clinical \& metabolic profile of various forms of Neurometabolic disorders in Bangladesh. It was conducted in the Department of Pediatric Neurology, from July 2017 to June 2020 in Bangabandhu Sheikh Mujib Medical University (BSMMU), Dhaka.

Children ( $<10$ years) clinically suspected Neurometabolic disorders were enrolled consecutively in this study. Total 59 children of neurometabolic disorders were included in this study at first on the basis of high index of clinical suspicion. Then diagnosis of forty-one (41) neurometabolic disorders was confirmed by biochemical test, neuroimaging study and sometimes molecular genetic testing. Demographic characteristics, age at onset of disease, clinical presentations, prenatal, natal, and postnatal history, family history, and parental consanguinity were recorded. Informed written consent was taken from their legal guardian or caregiver. Data were collected from their parents in structured predesigned questionnaire. Data analysis was performed by Statistical Package for Social Science (SPSS), version-22. Results were presented as text and tables.

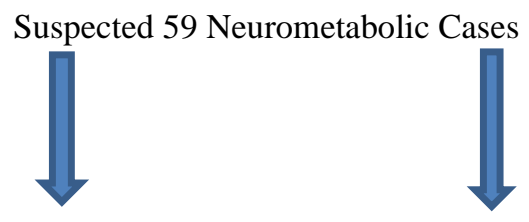

41confirmed cases were included
Excluded 18 cases due to incomplete investigation

\section{RESULT}

Among 59 suspected patients, total 41 patients were diagnosed as Neurometabolic disease. Most of the (63.2\%) patients were within 0-3 years age. Male patients $(70.73 \%)$ were outnumbered than female (29.26\%). Male: Female were 2.4:1. More than half of patients $25(60.90 \%)$ had parental consanguinity followed by Sib death 7 (17.07\%), Prematurity 11(26.82\%). Common Neurometabolic Diseases were aminoacidopathy $14(34.14 \%)$ and lysosomal storage disorders $15(36.58 \%)$ followed by mitrochrondrial disorders $5(12.19 \%)$, Urea cycle disorders 3(7.31\%), CHO metabolism disorders 5(2.43\%), Peroxismal disorders 3(7.31\%). Among aminoacidopathy, Organic acidamia 4(9.72\%), MSUD $3(7.31 \%)$, PKU 2(4.86\%), Gluteric Aciduria 3(7.31\%) and Biotinidase deficiency disorder $1(2.43 \%)$ were most common disorders. Among lysosomal storage disorders, mucopolysaccaridosis 4(9.72\%) and metachromatic leukodystrophy $8(19.44 \%)$ were most common disorders. Common presentation was developmental delay 37(90.24\%), developmental regression 21(51.21\%), hypotonia 29(70.73) and seizures $26(63.41 \%)$.

Neuroimaging changes found in about three-forth $(80.49 \%)$ of cases. About one-fourth $26.82 \%$ cases presented with white matter hyper intensity followed by basal ganglia hyper intensity $(19.51 \%)$ and Cerebral atrophy (14.63\%). Abnormally high ammonia and lactate found in 6(14.63\%) and $10(24.40 \%)$ cases respectively followed by Positive urinary ketones8 $(19.51 \%)$.

\begin{tabular}{ccc}
\multicolumn{3}{c}{ TABLE I: DISTRIBUTION OF PATIENTS BY AGE $(\mathrm{N}=41)$} \\
\hline Age & Number & Percentage $(\%)$ \\
\hline 0-3 years & 26 & 63.41 \\
3-6 years & 12 & 29.26 \\
6-12 years & 3 & 7.31 \\
Total & 41 & 100 \\
\hline
\end{tabular}

Table I showed the distribution of patient by age. Most of the $26(63.41 \%)$ children were within 1 day-3 years of age.

\begin{tabular}{ccc}
\multicolumn{3}{c}{ TABLE II: DistRIBUTION OF PATIENT BY SEX $(\mathrm{N}=41)$} \\
\hline Sex & Number & Percentage $(\%)$ \\
\hline Male & 29 & 70.73 \\
Female & 12 & 29.26 \\
\hline
\end{tabular}

Table II showed the sex distribution. Here male (70.73\%) is outnumbered than female $(29.26 \%)$. Male: Female were 2.4:1.

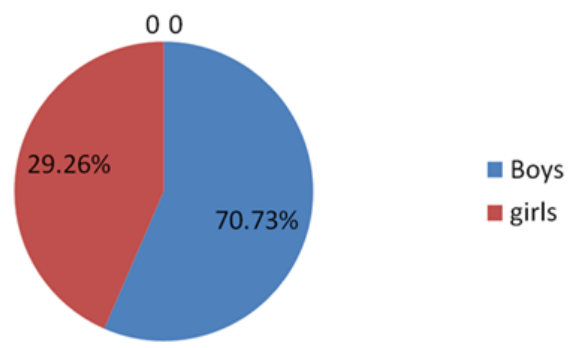

Fig. 1. Pie chart showed male (70.73\%) and female (29.26\%).

TABLE III: DISTRIBUTION OF PATIENT BY FAMILY HISTORY

\begin{tabular}{ccc}
\hline History & Number & Percentage $(\%)$ \\
\hline Consanguinity & 25 & 60.90 \\
Sib-death & 7 & 17.07 \\
Prematurity & 11 & 26.82 \\
\hline
\end{tabular}


Table III showed the Family History. About one -fourth of patients $25(60.90 \%)$ had parental consanguinity followed by Sib death 7 (17.07\%), Prematurity 11(26.82\%).

TABLE IV: DISTRIBUTION OF STUDY POPULATIONS BY TYPE OF NEUROMETABOLIC DISORDERS ( $\mathrm{N}=41)$

\begin{tabular}{ccc}
\hline \multicolumn{1}{c}{ Type } & Number & $\begin{array}{c}\text { Percentage } \\
(\%)\end{array}$ \\
\hline Amino acid disorder: & 14 & 34.14 \\
$\bullet \quad$ Maple Syrup Urine Disorder & 3 & 7.31 \\
• Phenylketonuria & 2 & 4.86 \\
• Glycine encephalopathy & 1 & 2.43 \\
• Glutaric aciduria & 3 & 7.31 \\
• Organic academia & 4 & 9.72 \\
- Biotinidase deficiency disorder & 1 & 2.43 \\
Urine cycle disorder: & 3 & 7.31 \\
Mitochondrial disorder: & 5 & 12.19 \\
CHO Metabolism disorder: & & \\
(Pompe-1) & 1 & 2.43 \\
Peroxisomal disorders: & & \\
(ALD-3) & 3 & 7.31 \\
Lysosomal Storage disorder: & & \\
$\bullet \quad$ Metachromatic leukodystrophy & 8 & 19.44 \\
$\bullet \quad$ Fucosidosis & 1 & 2.43 \\
• Mucopolisacharoidosis(MPS) & 4 & 9.72 \\
• Niemann Pick Disease & 1 & 2.43 \\
• Mucolipidosis & 1 & 2.43 \\
\hline
\end{tabular}

Table IV showed distribution of study populations by Type of Neurometabolic Diseases. Here, Common Neurometabolic Disorders were amino acid disorders 14(34.14\%) and lysosomal storage disorders $15(36.58 \%)$ followed by mitochondrial disorders 5(12.19\%), Urea cycle disorders $3(7.31 \%)$, CHO metabolism disorders 5(2.43\%), Peroxisomal disorders 3(7.31\%). Among amino acid disorders, Organic acidemia 4(9.72\%), MSUD 3(7.31\%), PKU 2(4.86\%), Glutaric aciduria 3(7.31\%), and Biotinidase deficiency disorder $1(2.43 \%)$ were most common disorders. Among lysosomal storage disorders, Mucopolysaccharidosis 4(9.72\%) and Metachromatic leukodystrophy 8(19.44\%) were most common disorders.

\section{Distribution of different types of neurometabolic disorder}

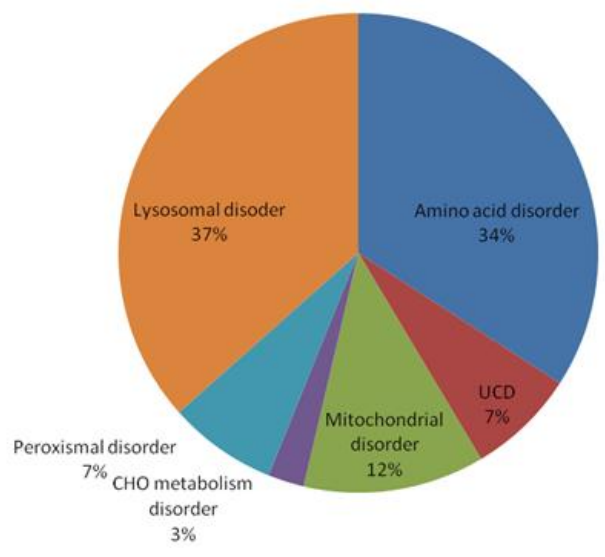

Fig. 2. Pie diagram of types of NMD.

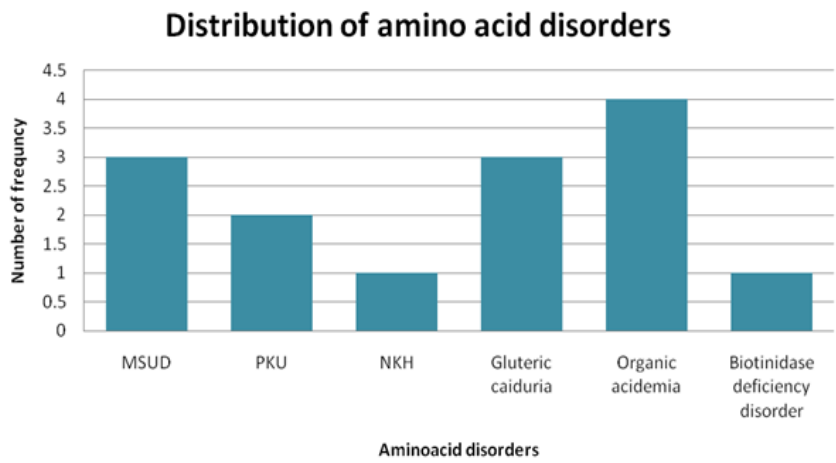

Fig. 3. Bar diagram of distribution of different types of amino acid disorders.

\section{Distribution of lysosomal storage disrders}

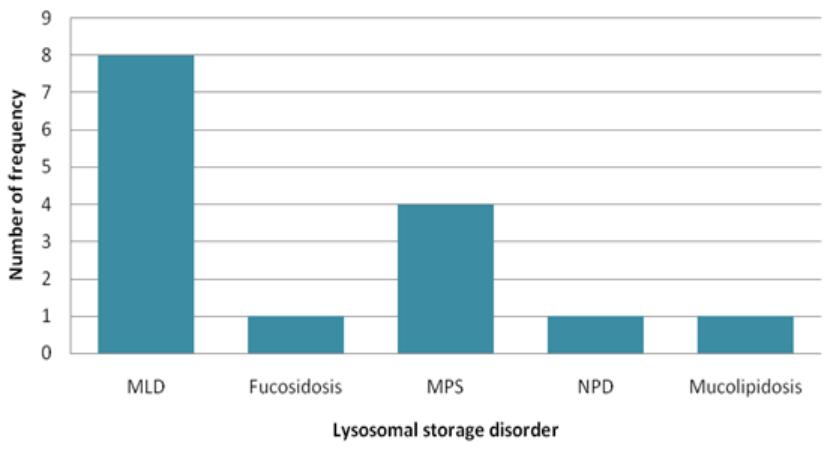

Fig. 4. Bar diagram of distribution of different types of Lysosomal storage disorders.

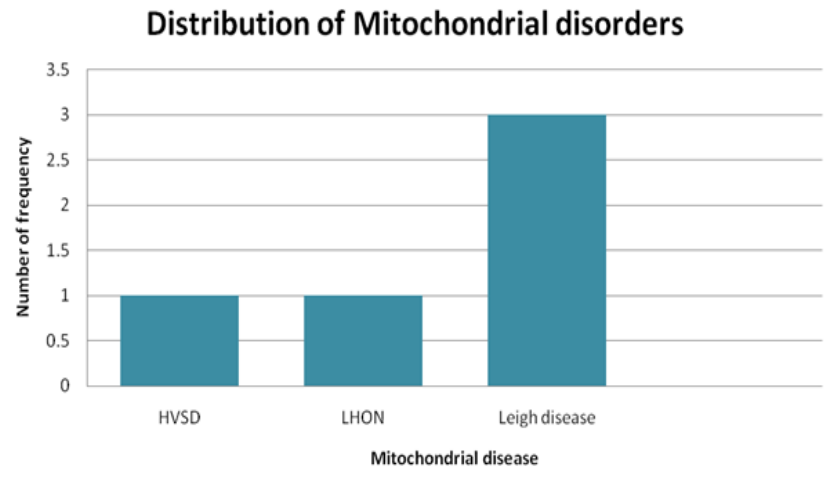

Fig. 5. Bar diagram of distribution of different types of Mitochondrial disorders

TABLE V: Distribution of Study Populations by PRESENTATIONS

\begin{tabular}{ccc}
\multicolumn{3}{c}{$(\mathrm{N}=41)$} \\
Presentation & Number & $\begin{array}{c}\text { Percentage } \\
(\%)\end{array}$ \\
\hline Developmental delay & 37 & 90.24 \\
Developmental Regression & 21 & 51.21 \\
Seizures & 26 & 63.41 \\
Failure to Thrive & 8 & 19.51 \\
Abnormal odor in urine & 6 & 14.63 \\
Facial dysmorphism & 5 & 12.19 \\
Organomegaly & 5 & 12.19 \\
Hypotonia & 29 & 70.73 \\
Vomiting & 6 & 14.63 \\
Unconsciousness & 5 & 12.19 \\
\hline
\end{tabular}

Table V showed distribution of study populations by presentation. Common presentations were developmental delay 37(90.24\%), developmental regression 21(51.21\%), hypotonia $29(70.73 \%$ ) and seizures $26(63.41 \%)$. 
TABLE IV: DISTRIBUTION OF STUDY POPULATIONS BY NEUROIMAGING

\begin{tabular}{lll}
\multicolumn{3}{c}{ CHANGES $(\mathrm{N}=41)$} \\
\hline MRI Changes & Number & Percentage $(\%)$ \\
\hline White matter Hyper intensity & 11 & 26.82 \\
Bats wings appearance & 3 & 7.31 \\
Basal ganglia hyper intensity & 8 & 19.51 \\
Cerebral oedema & 1 & 2.43 \\
Cerebral atrophy & 6 & 14.63 \\
CCA & 4 & 9.75 \\
Normal & 8 & 19.51 \\
\hline
\end{tabular}

Table VI showed neuroimaging changes of study cases. Neuroimaging changes found in majority (80.49\%) of cases. About one-fourth $(26.82 \%)$ cases presented with white matter hyper intensity followed by basal ganglia hyper intensity $(19.51 \%)$, Bats wings appearance $(7.31 \%)$, Cerebral atrophy (14.63\%) and CCA (9.75\%).

TABLE VII: Distribution of StUdy Populations by Metabolic TeST

\begin{tabular}{ccc} 
& $(\mathrm{N}=41)$ & \\
\hline Metabolites & Number & Percentage $(\%)$ \\
\hline Amonia & 6 & 14.63 \\
Lactate & 10 & 24.40 \\
Positive urinary ketones & 8 & 19.51 \\
Low blood glucose & 3 & 7.31 \\
Biotinidase deficiency & 1 & 2.43 \\
Acylcarnitine deficiency & 5 & 12.19 \\
Urinary organic acids & 4 & 9.75 \\
MPS spot urine test & 4 & 9.75 \\
\hline
\end{tabular}

Table VII showed metabolic screen of the studied cases. Abnormally high ammonia and lactate found in 6(14.63\%) and $10(24.40 \%)$ cases respectively followed by Positive urinary ketones 8(19.51\%), Acylcarnitine deficiency $5(12.19 \%)$, Urinary organic acids $4(9.75 \%)$ and MPS spot urine test $4(9.75 \%)$.

\section{DISCUSSION}

Neurometabolic disorders are inborn errors of metabolism with neurological abnormalities. These disorders, an inherited conditions are caused by lack or dysfunction of an enzymes or vitamins necessary for a specific chemical reaction in the body [1]. Over one third of the inherited metabolic disorders are characterized by the central nervous system involvement [2]. Presentations are usually variable depending on the age of onset. Disorders are usually grouped according to age such as infancy (1-12 months), late infantile/early juvenile onset period (1-5 years), early juvenile and late childhood period (5-15 years).In this study $26(63.41 \%)$ children were found within 1 day-3 year of age and male: female were found $2.4: 1$. It is consistent with a previous study where $53 \%$ of children were found to be within 1-5 years of age and male were also found predominantly affected (64\%) [6]. As most of the Neurometabolic disorders are transmitted by autosomal recessive inheritances, background family history is an important clinical indicator for suspicion of Neurometabolic disease. In a previous study $31 \%$ patient had history of parental consanguinity and 5\% had affected sib [6]. In this study about one -fourth of patients $25(60.90 \%)$ had parental consanguinity, sib death found in $7(17.07 \%)$ and prematurity in $11(26.82 \%)$. In Egypt, inborn errors of metabolism are common, presumably because of the high rates of consanguinity (38\%) [7]. Presentation is heterogeneous in
Neurometabolic disease. Clinical symptoms depend on the particular metabolic pathway that is involved. Clinical manifestations range from antenatal onset disorders with malformations, fulminant neonatal encephalopathy, to later onset and milder phenotypes. Clinical course may vary with relapsing and remitting clinical manifestations, step ladder pattern of worsening, chronic progressive course, and also spontaneous improvement of various clinical manifestations [8]. In this study common presentation were developmental delay $37(90.24 \%)$, developmental regression $21(51.21 \%)$, hypotonia $29(70.73 \%$ ) and seizures $26(63.41 \%)$. Abnormal body and urinary odor are an important diagnostic indicator, which often usually give specific diagnostic clue. Such as inglutaric acidemia (type II): sweaty feet, 3-Hydroxy-3methylglutaric aciduria: cat urine, Isovaleric acidemia: sweaty feet, Maple syrup urine disease: maple syrup, Hypermethioninemia: boiled cabbage, Multiple carboxylase deficiency: tomcat urine, Phenylketonuria: mousy or musty Tyrosinemia: boiled cabbage, rancid butter [8]. In this study abnormal urine odor found in $14.63 \%$ of patients. The pattern of involvement in the brain offers clues to the possible underlying metabolic derangement; some of which are pathognomonic, some suggestive, and others non-specific. The pattern of involvement depends on the stage of brain development and myelin maturation, \& some other factors like receptor and neurotransmitter distribution, cell and region-specific variability in molecular composition, energy requirements and affinity to certain toxins [9]. In this study neuroimaging changes found in majority $(80.49 \%)$ of cases. About one-fourth $(26.82 \%)$ cases presented with white matter hyper intensity followed by basal ganglia hyper intensity $(19.51 \%)$, bats wings appearance $(7.31 \%)$, cerebral atrophy $(14.63 \%)$ and CCA $(9.75 \%)$. Though earlier recognition of inborn errors of metabolism has the potential to reduce morbidity and mortality rates specific diagnosis is often challenging due to lack of metabolic testing facilities in developing countries. Elevation of blood ammonia is usually caused by defects of urea cycle enzymes. Infants with elevated blood ammonia levels from urea cycle defects commonly have normal serum $\mathrm{pH}$ and bicarbonate values. Elevation of ammonia is also observed in some infants with certain organic acidemias Elevated lactate along with ketoacidosis with or without hyperammonemia's seen in lactic acidosis. Ketoacidosis with or without elevated lactate along with hyperammonemia points towards organic aciduria [10]. Other investigations can be done according to clinical suspicion and finally confirmed by genetic testing. In this study abnormally high ammonia and lactate found in $6(14.63 \%)$ and $10(24.40 \%)$ cases respectively followed by positive urinary ketones $8(19.51 \%)$, Acylcarnitine deficiency $5(12.19 \%)$, Urinary organic acids $4(9.75 \%)$ and MPS spot urine test $4(9.75 \%)$.

In this study with 213 children of neurometabolic diseases 34 different neurometabolic disorders were diagnosed and classified in the 7 sub classes, consisting of: organic acidemia and aminoacidopathy (122 patients), storage disease (37 patients), leukodystrophy (27 patients), other classes consisted of: lipid oxidation disorders, urea cycle disorders, progressive myoclonic epilepsy; and peroxisomal disorders (27 patients). They suspected neurometabolic disorders in patients with developmental delay or regression, with or 
without seizure, abnormal neurologic exam along with positive family history of similar disorder, abnormal brain imaging with specific patterns11. In another study from Bangladesh 128 children of suspected neurometabolic disease, showed parents of $39(31 \%)$ had history of consanguineous marriage, seizure was present in $96(75 \%)$ children and abnormal EEG found in 83 (65\%). Plasma ammonia and lactate was raised in $53(54 \%)$ and $40(43 \%)$ respectively. TMS was abnormal in $70(63 \%)$ cases and decreased serum biotinidase activity found in 17(68\%) cases6. Results of previously found studies were consistent with our findings.

Finally, from all clinical, biochemical, and imaging findings, amino acid disorders 14(34.14\%) and lysosomal storage disorders $15(36.58 \%)$ followed by mitochondrial disorders $5(12.19 \%)$, Urea cycle disorders $3(7.31 \%)$, CHO metabolism disorders $5(2.43 \%)$, Peroxisomal disorders $3(7.31 \%)$ were found in this study. Among aminoacid disorders, Organic acidemia 4(9.72\%), MSUD 3(7.31\%), PKU 2(4.86\%), Glutaric aciduria 3(7.31\%), and biotinidase deficiency disorder $1(2.43 \%)$ were most common disorders. Among lysosomal storage disorders, Mucopolysaccharidosis 4(9.72\%) and Metachromatic leukodystrophy $8(19.44 \%)$ were most common disorders.

\section{CONCLUSION}

In this study commonest neurometabolic disorders were aminoaciduria and lysosomal storage disease. Among Amino acid disorders, Organic acidemia, MSUD, Glutaric aciduria and in Lysosomal storage disorders, metachromatic leukodystrophy \& mucopolysaccharidosis were commonest disorders. Common presentations were developmental delay, developmental regression, and seizure disorders. Neuroimaging changes were found in about three- forth cases in this study.

\section{AUTHORS CONTRIBUTION}

Dr. Gopen Kumar Kundu, Dr. Rumana Islam participated in the development of protocol and the drafting of the manuscript. Dr. Sanjida Ahmed participated in the development of reviewing of the manuscript.

\section{ACKNOWLEDGMENT}

We are grateful to patients and their families with neurometabolic disorders for focused discussion in this paper and for their encouragement.

\section{REFERENCES}

[1] Karimzadeh P. Approach to neurometabolic diseases from a pediatric neurological point of view. Iranian journal of child neurology. 2015;9(1):1.

[2] Christopher R, Sankaran BP. An insight into the biochemistry of inborn errors of metabolism for a clinical neurologist. Annals of Indian Academy of Neurology. 2008 Apr;11(2):68.

[3] Sharma S, Kumar P, Agarwal R, Kabra M, Deorari AK, Paul VK. Approach to inborn errors of metabolism presenting in the neonate. The Indian Journal of Pediatrics. 2008 Mar;75(3):271-6.

[4] Aicardi J, Ogier H. Metabolic Diseases. In: Aicardi J, Diseases of the Nervous System in Childhood, 3rd ed. London, Mc Keith Press. 2009. p 243-326.

[5] Biswas A, Malhotra M, Mankad K, Carney O, D’Arco F, Muthusamy K, Sudhakar SV. Clinico-radiological phenotyping and diagnostic pathways in childhood neurometabolic disorders - a practical introductory guide. Translational Pediatrics. 2021 Apr;10(4):1201.

[6] Mahbub M, AzamAM, Mazumder SC, Debnath B, Khan NZ Background History, Clinical Presentation and Laboratory Profile in Cases of Suspected Neurometabolic Disorders.Bangladesh Journal of Child Health. 2015;39(1):24-9.

[7] Temtamy SA, Kandil MR, Demerdash AM, Hassan WA, Meguid NA, Afifi HH. An epidemiological/genetic study of mental subnormality in Assiut Governorate, Egypt.Clinical genetics. 1994 Nov;46(5):347-51.

[8] Biswas A, Malhotra M, Mankad K, Carney O, D’Arco F, Muthusamy K, Sudhakar SV. Clinico-radiological phenotyping and diagnostic pathways in childhood neurometabolic disorders - a practical introductory guide. Translational Pediatrics. 2021 Apr;10(4):1201

[9] Van der Knaap MS, Valk J. Magnetic resonance of myelination and myelin disorders. Springer Science \& Business Media; 2005 Dec 5.

[10] Gulati S, Vaswani M, Kalra V, Kabra M, Kaur M. An approach to neurometabolic disorders by a simple metabolic screen. Indian pediatrics. 2000 Jan 1;37(1):63-8.

[11] Karimzadeh P, Jafari N, Biglari HN, Jabbehdari S, Zadeh SK, Abadi FA, Lotfi A. Neurometabolic diagnosis in children who referred as neurodevelopmental delay (a practical criteria, in Iranian pediatric patients). Iranian journal of child neurology. 2016;10(3):73. 